\title{
Parameter tuning of the HCSCROCFO-3Opt algorithm for solving the capacitated vehicle routing problem
}

\author{
Teerapun Saeheaw ${ }^{a^{*}}$
}

${ }^{a}$ Department of Teacher Training in Mechanical Engineering, Faculty of Technical Education, King Mongkut's University of Technology North Bangkok, Bangkok, Thailand

\begin{tabular}{l}
\hline C H R O N I C L E \\
\hline Article history: \\
Received February 202020 \\
Received in Revised Format \\
May 232020 \\
Accepted June 232020 \\
Available online \\
June, 23 2020 \\
\hline Keywords: \\
Capacitated vehicle routing \\
problem \\
Cuckoo search \\
Central force optimization \\
Chemical reaction optimization \\
3-opt
\end{tabular}
\begin{abstract}
A B S T R A C T
This paper proposes the cuckoo search (CS), central force optimization (CFO), chemical reaction optimization (CRO) and 3-Opt for solving the capacitated vehicle routing problem (CVRP). HCSCROCFO-3Opt, which is the parallel hybrid algorithm that is proposed, is a form of augmented HCSCROCFO with a local search process founded on CS that utilizes positive aspects of the other optimization approaches including CRO and CFO in order to enhance quality of initial population and improve local search, correspondingly. The work is motivated by the need to enhance the computational effectiveness through attainment of improved outcomes compared to previous popular solutions, to explore the features of different parameters of to seek some ideal solutions. The first stage entails solving of CVRP through setting a variety of values to tune parameters for the HCSCROCFO-3Opt proposed. Then initialization of algorithm CS, $\mathrm{CRO}, \mathrm{CFO}$ parameters are accomplished through tuning parameters within a tuning cycle. Subsequently, a novel solution is swapped in a random manner through a levy flight within the central loop, followed by execution of the hybrid solution as well as new CRO, CFO and CS algorithm solutions, whose implementation is supposed to enhance results for the local 3-Opt. Ultimately, the most ideal solution for general hybrid model's solution space is identified, after which the solution that is best-suited for the CVRP purposes is presented. Within the standard CVRP cases, reported computational tests in large scale in the literature demonstrate the efficiency of presented approach.
\end{abstract}

\section{Introduction}

The capacitated vehicle routing problem (CVRP), which originates from the vehicle routing problem (VRP), can be categorized as a conventional combinatorial optimization challenge. It was established in late 1958, and continues to be a common subject in operations research environments (Dantzig \& Ramser, 1959). Within the framework, the satisfied minimal costs emanate from the route used in dispatching the vehicle fleets from a central node or depot, where the capacity of every vehicle is uniform, and all customers have specific demands. In most cases, the cost differs based on the number of vehicles used, duration of travel, distance, or a blend of these aspects. From a practical perspective, the fundamental formulation of the VRP challenge is influenced by issues like capacity of the vehicle or time interval for serving a customer, which creates the CVRP (Belfiore et al., 2008). The meta-heuristic approach was created from frameworks at a higher level to facilitate efficient and effective exploration of a search space developed in the past three decades.

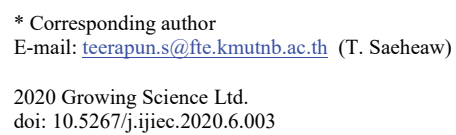


The approach is a novel form of combined algorithm merging simple heuristic approaches. Metaheuristic methods are synonymous with efficiency in regards to avoiding local optima associated with the problem solution-space. The most ideal group processes for addressing issues of combinatorial optimization may be derived from the meta-heuristic approach. Different adaptations, additions and blend of the fundamental movements for various forms of VRP are presented in the sections that follow (Vidal et al., 2012). The local search utilized in enhancing heuristics from addressing a VRP is the first solution with multiple adjacent frameworks, along with a best-accept approach. At the local level, the search processes are utilized in general method to address challenging issues of combinatorial optimization. Majority of used neighbourhoods are common and have been introduced previously in the framework of different constructions. There is a possibility of moving or swapping a VRP instance in a sequence characterized by successive vertices, instead of one vertex, with the adjacent operators such as Or-opt, and 3-opt as special instances. Generally ideal solutions were also commonly observed through the use of simple random 2-opt move and 3-opt perturbations of move (Blazinskas \& Misevicius, 2011). Metaheuristic hybrids are typically explored for combinatorial optimization that emphasizes on convergent processes (Ólafsson, 2006). For this paper, the proposed method is utilized in addressing the CVRP case with HCSCROCFO-3Opt; nevertheless, it may also be utilized for other system classes. Similar to other common existing meta-heuristics founded on population such as ant colony optimization (ACO), simulated annealing (SA0, artificial immune system (AIS), firefly algorithm (FA), artificial bee colony $(\mathrm{ABC})$, differential evolution (DE), particle swarm optimization (PSO0 and genetic algorithm (GA) along with others approaches leveraging on similar methodologies and approaches, their key aspects entail mirroring the ideal of intelligence inspired by nature through leveraging the capacity of innate parallel stochastic search aspects. PSO and CFO are similar, however, CFO is also exceptional in regards to its deterministic characteristics, attaining faster convergence to universal extreme, along with its gravitational relations. CRO resembles GA; nonetheless, its size of population includes various reactants differing between generations, while the setting of the population size in GA is constant. CS closely resembles DE; nevertheless, it uses some kind of elitism in selecting, in a manner that is similar to the harmony search. In comparison to other meta-heuristics, CS has a constant fine balance of intensification and randomization with less fine-tuned considerations. Therefore, it is simpler to address issues associated with optimization in an efficient manner.

The structure of the remainder of the paper is structured as presented here. The second part presents the problem. The third part presents a description of the meta-heuristic approaches. The fourth part explores the hybrid methodology. The firth part provides a presentation of the computational experiment. Ultimately, the sixth section presents a summarization of the conclusions.

\section{Problem Description}

As the conventional version of the vehicle routing problem (VRP), the CVRP seeks to identify the least aggregate cost routes for a homogenous fleet route serving a collection of customers. The ultimate aim is identification of a collection of routes minimizing the number of utilized units as well as the vehicles' constraint capacity (Jin et al., 2014). The optimization of the CVRP aims at identifying a collection of routes that has the lowest aggregate costs beginning and ending at the depot. In the depot, every vertex is visited once, and the distance of every route should be longer or equal to the least distance for every route. The lowest collection of vehicles as well as the load capacity should be determined. Form a mathematical perspective, $G=(V, E)$ represents the collection of clients, depot along with their associations as modeled in a graphical manner. The graph may be completed with vertices $v=\{0,1, \ldots, k\}$, where the depot is represented by the vertex $\{0\}$ and the rest denote the customers. All the edges are described as $e_{i j}=\{i, j\} \in E$. Edges have linked value $c_{i j}$ representing the positive cost of transportation, and every client $i \in=V^{\prime}=V \backslash\{0\}$ is linked to demand $d_{i} . S=(1,2, \ldots, m)$ represents a collection of homogenous transportation means whose capacity is $Q$. The depot must satisfy the collection of demands from every customer. The CVRP is comprised of construction of a collection of a maximum of routes, where (1) each of them begins 
and culminates at the depot; every demand is satisfied; (3) they do not exceed the capacity of the vehicles; (4) only one vehicle visits a customer; and (5) the cumulative cost is reduced to a minimum. Mathematically, the formulas are described as shown below:

$n=$ the number of nodes for each instance,

$m=$ the number of vehicles used for each instance,

$x_{i j}= \begin{cases}1 & \text { if the vehicle travels directly from node } i \text { to node } j \\ 0 & \text { otherwise. }\end{cases}$

$y_{i k}=\left\{\begin{array}{l}1 \quad \text { if the node } i \text { is visited by the vehicle } k \\ 0 \quad \text { otherwise. }\end{array}\right.$

The CVRP's integer programming (IP) formulation can thus be presented as shown below:

$$
\begin{array}{lll}
\min & & \\
& \sum_{i=0}^{n} \sum_{j=0}^{n} c_{i j} x_{i j} & \\
\text { subject to: } & \sum_{i=0}^{n} x_{i j}=1 & \\
& \sum_{i=0}^{n} x_{i 0}=m & \\
& \sum_{j=0}^{n} x_{i j}=1 & \\
& \sum_{j=0}^{n} x_{0 j}=m & \\
& \sum_{i=0}^{n} q_{i} y_{i k} \leq Q & k=1, \ldots, n \\
& \sum_{i \in S} \sum_{j \in N-S} x_{i j} \geq 1 & \phi \neq S \subset\{1, \ldots, n\},|S| \geq 2 \\
& \sum_{i=N-S} \sum_{j \in S} x_{i j} \geq 1 & \phi \neq S \subset\{1, \ldots, n\},|S| \geq 2 \\
& x_{i j} \in\{0,1\} & i=0, \ldots, n ; j=0, \ldots, n \\
& y_{i k} \in\{0,1\} & i=0, \ldots, n ; k=0, \ldots, m
\end{array}
$$

The first function is used in minimizing the cumulative distance covered in a trip. The second and third constraint equations guarantee that every vehicle visits every node for one instance and the depot is visited $\mathrm{m}$ times. The fourth and fifth constraint equations make sure that each node is left by a vehicle only one time and the depot is left m number of times. The sixth control equation inhibits loading of vehicles in a manner that exceeds their capacity. The seventh and eight constraint equations avert the occurrence of sub-tours for every vehicle. Ultimately, the ninth and tenth constraint sets create the binary situation descriptions of the variables.

\section{Meta-heuristic Algorithms}

In this paper, a comparative assessment of four meta-heuristic procedures for addressing optimization of chemical reaction, optimization of central force, CVRP-cuckoo search and 3-opt is presented. It goes further to conduct an analysis of how they perform in regards to quality of solution and efficiency. 


\subsection{Cuckoo Search Algorithm}

Cuckoo Search (CS) is a natural meta-heuristic search procedure that was established in 2009 by Yang and Deb. The inspiration for the procedure emanates from reproduction of cuckoo strategy that may lay eggs nests belonging to other birds, regardless of their species. When the host bird discovers that the eggs are foreign, it may decide to destroy them or abandon the nest together with the eggs. This has created a situation where cuckoo eggs have evolved to become similar to eggs belonging to birds hosting nests locally (Yang \& Deb, 2009; Zhou et al., 2013). A random walk, characterized by a Levy co-efficient and exponent is used in determining cuckoos. In this manner, Levy flights for random walks can be implemented in a simple manner through Mantegna's Algorithm. Paul Pierre Lévy (1886-1971) developed the Lévy Stable Probability Laws. Lévy flights (LFs) can be defined as non-Brownian random walks that include long jumps (physics, nature, finance). From a mathematical perspective, they can be considered as random processes of an alpha-stable Levy nature. Levy motion and the distribution ranges are $0<\alpha \leq 2,-1 \leq \beta \leq 1,0<\gamma<\infty$, and $-\infty<\Delta<\infty$ (Yang, 2010). CS utilizes Lévy flights to make long jumps/steps for random walks, which may be as a result of the large step Levy distribution, which has endless variance and mean. In this case, the successive cuckoo jumps/steps basically create a process of random walk that abides by a distribution of power-law step-length whose end is heavier. A number of novel solutions must be created through a Levy walk in regards to the most ideal solution attained so far; in order to increase the rate of local search. The CS randomization is a procedure founded on the population. It is vital since the step length is heavier at the end, and there is a possibility of large steps. $\mathrm{CS}$ is a form of meta-population procedure, which restricts the number of aspects to remain lower than other procedures. Every nest may therefore denote a collection of clarifications, which may be protracted (Yang and Deb, 2009). The number of the host nest remains constant, and the probability of the laid egg being found is $p_{a} \in[0,1]$. The egg may be discarded or the nest abandoned so that the host bird can construct a totally new nest. Conversely, while implementing the CS algorithm, only two aspects must be considered: the size of the population $\mathrm{n}$, and pa. It is possible to approximate the last assumption by the portion pa of the nests $\mathrm{n}$ that have been substituted by other nests that denote random results. The efficiencies strategy to identifying the universal optima has a bigger rate of success rate and a fine balance of intensification and randomization, as well as lower number of control aspects.

\subsection{Central Force Optimization}

The recently developed multidimensional deterministic search meta-heuristic, which rests on the analogy of gravitational kinetics, is the central force optimization, also acronymic as CFO. The CFO algorithm model is represented by the probes whereby the algorithm flies through the decision space by analogy to the masses that are moved by gravitational force. They are used in the detection and location of the objective function's maximum value (Formato, 2007). There is a similarity between the computed results of the central force optimization and those of the plots linked to gravitational trapping of near-earth objects, which suggests that there is a possibility of the existence of another universal "CFO hyperspace directional derivative." Trapping, which is deterministic algorithm, is the greatest challenge with the CFO. The measures for selection running aimed at averting the local trapping are thus sufficient, but again not a necessity for trapping, especially in osclillation of the average distance curve. The elimination of the trapping is possible with this feature. The CFO relies on the following seven user-specified parameters to run; $N_{t}, N_{p}, G, \alpha, \beta, \Delta t$, and $F_{\text {rep. }}$. The way an individual selects these parameters can allow for them to get a number of global optimizations. In addition to that, a careful selection of the run parameters can allow for an individual to get the best out of the CFO's features as well as its simplicity, ability to solve the problem and efficiency. Users can quickly specify suitable run features due to the CFO's deterministic feature. That is, the feature, together with its normally rapid convergence, albeit to a local maximum, may be of significance fir the users. The accurate parameters due to the CFO's gradient like nature implies that it can be run very fast, which allows for it to converge on utmost faster than other algorithms. One of the key elements of the CFO is its real-time parameter tuning. This is because; the running parameters may be altered in the behavioral response of the algorithms. A number of reported 
instances indicated that some theoreticians are aiming at coming up with new models both in antenna design engineering and optimization. Tuning, as one of the key parameters based on real time feedback is of significance owing to the fact that selection of a number of running optimization algorithm is subjective, and at the same time differs by the comprehension and skills of the user with certain algorithm. The highly variable's outcome is possibly obtained without a definitive assigned parameter method, and also run with similar issue.

\subsection{Chemical Reaction Optimization}

The stability of CFO reaching low energy state is as a result of its emulation of the molecule interactions in a chemical reaction ( $\mathrm{Lam} \& \mathrm{Li}, 2010$ ). This is as result of the molecular collisions with the walls of the container or with other similar molecules.

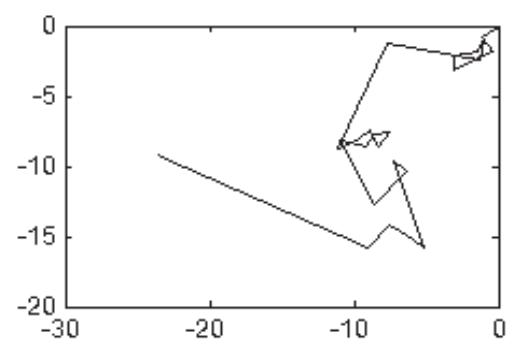

(a)

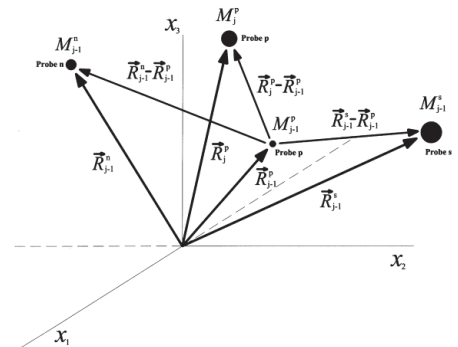

(b)

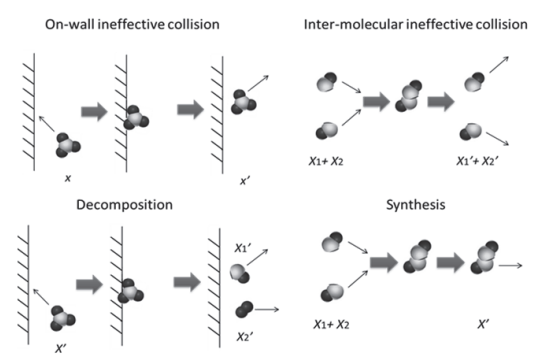

(c)

Fig. 1. The representation of search methods of representing algorithms (a) Levy flight distribution in CS (b) multi-dimensional search in CFO (c) four chemical reactions types in CRO

The four types of elementary reactions among molecules includes decomposition, synthesis, on-wall ineffective collision as well as inter-molecular ineffective collision. The on-wall ineffective collision only involves a single molecule with the container's walls. An intermolecular ineffective collision is a collision of more than one molecule while in decomposition; there is the production of numerous molecules from a single molecule. The synthesis as an elementary reaction between molecules involves a combination of more than one molecule combining to form a single molecule. Thus, in synthesis, the number of molecules is constant, but the original solutions in the neighborhoods are searched. The remaining two reactions generate new solutions that differ from the initial ones by the potential energy change which, in turn, assists the algorithm to jump out of the local optimums. The diversification can allow for the CRO to proceed with the search even in situations where for an extended period, the CRO fails to get a lesser energy state. The intermolecular ineffective as well as the on wall collisions allow for the intensification, while on the other hand, diversification is performed by synthesis coupled with decomposition synchronously. In attempting to redistribute the energies between the molecules, the system interchanges the energies from one to the other in different ways. It is at this section that the implementation through the elimination of chemical reaction system functions is described. The $P E$ is the potential energy, which is the objective function for measurement while the molecular structure is the answer to the problem, and $K E$ (kinetic energy) is the tolerance parameter for recording an inferior solution while on the other hand, the amount of hits is the current number of movements. In addition to that is the present optimum solution is represented by minimum structure while the minimum number is the present optimum rate. The current optimum solution is represented by the minimum hit number which is attained by the number of movements. Performing CRO is first done by having pop that is randomly generated through the popsize, number of solutions in the solution space. As a result, this action prompts the increase in the extent of the search over $f$. Thus, there is the attainment of the value of the point of integration iteration. The CRO iteration is selected through the collisions that mainly determine if it is an inter-molecular collision or uni-molecular collision. The results is a random value $t$ within the interval of 0,1 . In a situation where $t$ is greater than MoleColl, uni-molecular collision occurs. If not, an intermolecular collision occurs. The settings of the CRO measures are; MoleColl, PopSize, KELossRate, 
$\beta$, InitialKE and $\alpha$ (Lam et al., 2010). Various met-heuristic studies have made experimental designs of the parameter set, like the random selection of a suitable value of molecules from pop, however finding the right parameter for tuning is what has been termed as combinatorial optimization problem. Addressing the problem by utilizing the average parameter tuning problem over the meta-heuristic algorithm with local search may not be the appropriate approach. However, we utilized the design of experiment parameter set for the injection of the range numbers and values to the CRO. As such, we attempted to separate measurements with continuous domains from those parameters having discreet domains, at which every number or value in the domain is tried (Urli, 2015). The two main advantages of the CRO algorithm are escaping entrapment in a local optimum by the CRO and again, searching neighborhoods for the initial solution.

\subsection{3-Opt Local Search}

3-Opt, first coined by Bock in 1958, is a local search algorithm that plays an integral role in finding a solution to a traveling salesman's problem. The method eliminates the three segments of a path and then comes up with a new path using the remainder segments. One of the segments is cut down by the change that involves path direction. The path that is considered longer than the initial one is automatically rejected and the shorter one is accepted. Once the shortest path is obtained, the repetitive process comes to a close (Marlow et al., 2007).

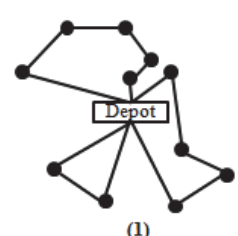

(1)

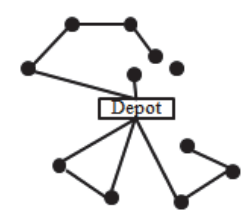

(2)

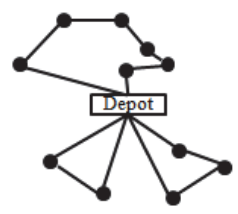

(3)

Fig. 2. 3-Opt local search. (1) The unimproved solution, (2) omitting three arcs, and (3) the improved solution

\section{Hybrid Methodology}

The amalgamation of meta-heuristic and other techniques for optimization results in the formation of the hybrid meta-heuristic method. The quality of provided solutions is improved by the hybrid meta-heuristic method. However, there is no study that has addressed the CVRP that has used simple random 3-opt using the HCSCROCFO. This study therefore tries to offer candidate ranking and listing techniques that promotes the ability of meta-heuristics hybridization with CRO, CFO and CS in solving CVRP. The basic hybridizations are conducted using the ILS (iterated local search). The ILS is a meta-heuristic that is based on a simple concept instead of repetitiveness of the local search in an attempt to achieve random starting solutions. The solutions, random incumbent that is done using the iterated local search results in starting solution that is used in the subsequent iterations. The practice is conducted with the aim that the randomization technique would achieve a local minimum solution (Blum et al., 2011). The ILS used in the 3-opt local search in the main structure of the hybridizations is implemented by this search process, together with the performance or the parallel meta-heuristic methodologies. In addition, the search, apart from the 3-opt local also implements the various exploration schemes that are utilized in the parallel search techniques. The proposed HCSCROCFO-3Opt's procedures are well explained in the pseudocode. The leading step is to read the CVRP instance to the variables, then setting the range of measures tuning values for hybrid, followed by looping the central cycle with parallel CFO, CRO and CS hybridization as you maintain the best of each solution already obtained. The final stage is finding the well-known solution.

\section{Computational Experiment}

Twelve examples from 3 categories of CVRP scales were chosen to be tested for efficiency, and with available techniques as well as verification of the validity of the techniques adopted in this paper. The first case was the Set A instances followed by Set $\mathrm{P}$ instances and as a final point, Set $\mathrm{E}$ instances. These 
problems have so far been utilized by a number of studies as point of reference to shed light on the efficiency of algorithms. Windows 10 platform's MATLAB R2018b within an Intel Core i7 CPU at 3.40 $\mathrm{GHz}$ with $16 \mathrm{~GB}$ of RAM were used for all computational experimentations.

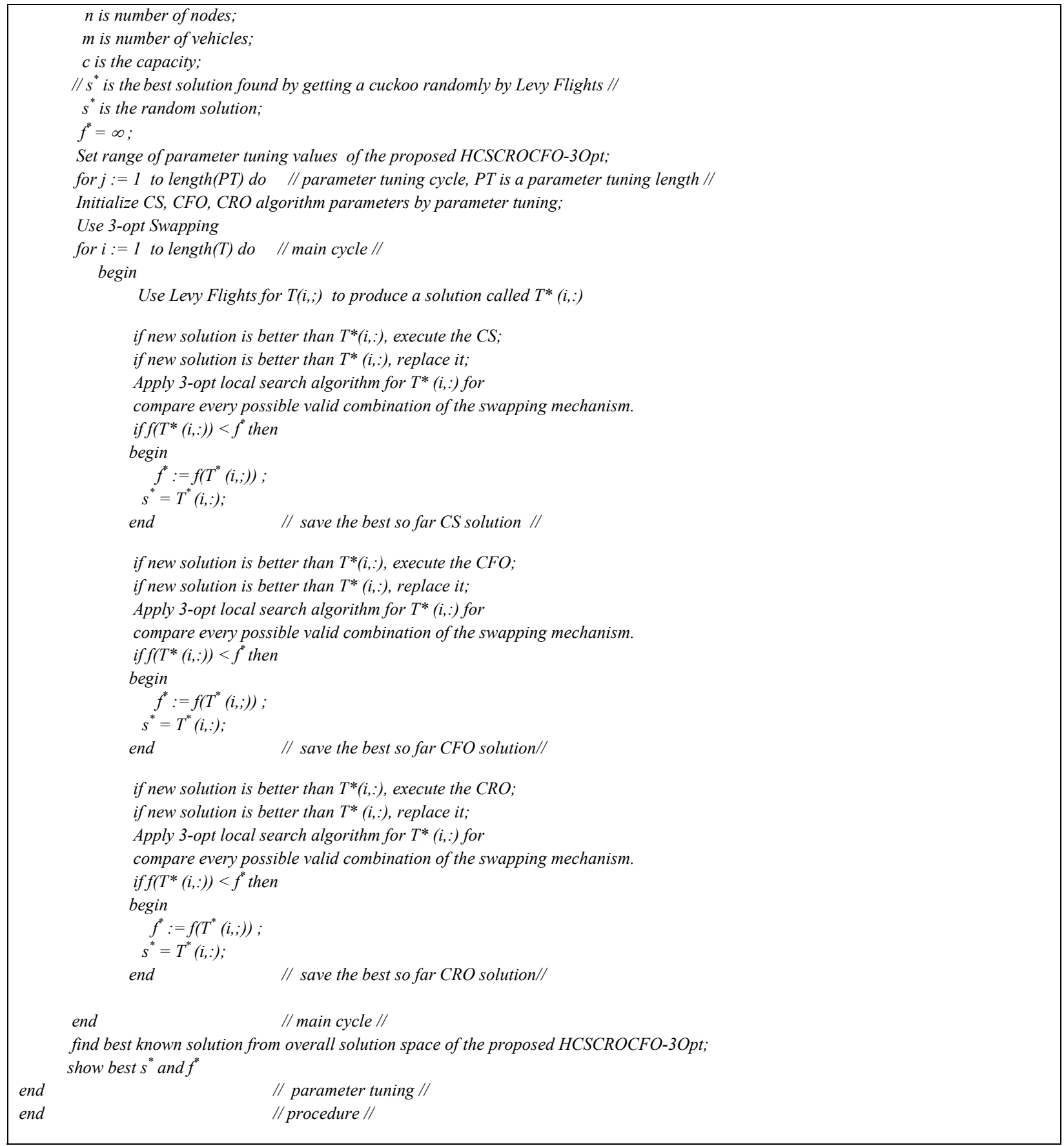

Fig. 3 shows the parameter tuning. The optimal parameters for the proposed HCSCROCFO-3Opt are shown for CS ( 0.7 for Alpha, 0.2 for Beta, 0.7 for Delta, and 0.5 for Gamma), CRO ( 9 for IKE, 9 for IPS, 1 for IBF, 10 for CRT, and 10 for LRT), and CFO ( 0.5 for Alpha, 0.7 for Beta). The replacement of the corresponding value with a new one is done subsequent to the validation that a fitting value is gotten for a particular parameter. After the replacement, the next step is to proceed to the next parameters with an amended combination. 


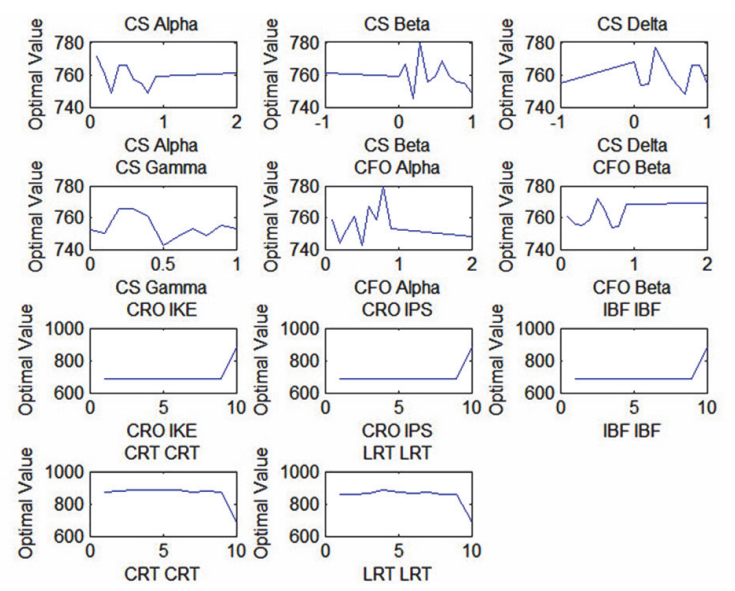

Fig. 3. Parameter tuning for the HCSCROCFO-3Opt

This study compares the proposed HCSCROCFO-3Opt presented in terms of average function evaluation, the average success rate and convergence profiles. A higher success rate can be achieved by the proposed HCSCROCFO-3Opt.The outcomes are tabulated in Table 1, where data about every case are found. The data are for; I_BKS a solution that is well known at the website http://vrp.galgos.inf.pucrio.br/index.php/en/; R_BSK that acts as a point of verification of real costs of the well-known solution as expounded by Juan et al. (2010); vehicle capacity, CWS that is in place of the costs resulting from the parallel version of heuristic (CWS); tightness (demand or capacity); SR-GCWS that is, the resultant solution from CVRP classical heuristic with the Monte Carlo replication model. Finally, the HBA-PR represents a hybrid bat algorithm with path relinking and as such, the HCSCROCFO-3OPT is the solution to the VPR cases identified in the ILS. The results from Table 1 written in bold font are those that are equal to the best known solution in the studies. In general, when compared to the traditional and new meta-heuristics for solving the CVRP, the proposed HCSCROCFO-3Opt matched with the best known solutions. Among the results from the simulation resulting from the test of HCSCROCFO-3Opt are that, the performance of HCSCROCFO-3Opt is prominent and also, there is a high level of efficiency when using proposed HCSCROCFO-3Opt to solve the CVRP. However, Table 2 indicates that there is no difference between the proposed HCSCROCFO-3Opt and other algorithms. The outcomes can end in the best solutions. Furthermore, the proposed algorithm finds the BKS for all of the instances tested. Trajectory techniques are found to be affective in the exploration of promising areas in the search space, while the identification of promising areas in the search space is done using population-based methods (Blum \& Roli, 2003). Therefore, the meta-heuristic hybrid is successful because they combine the trajectory and population-based methods techniques.

\section{Table 1}

Relates algorithms for capacitated VRP Illustrations. Augerat et al. Set A, E, and P instances are selected with the plan to test the practicality of the proposed HCSCROCFO-3Opt

\begin{tabular}{ccccccccc}
\hline Instance & Capacity & Tightness & I_BKS & R_BSK & CWS & SR-GCWS & HBA-PR & HCSCROCFO-3Opt \\
\hline A-n33-k5 & 100 & 0.89 & 661 & 662.76 & 712.05 & 662.11 & 662.1101 & $\mathbf{6 6 1}$ \\
A-n33-k6 & 100 & 0.9 & 742 & 742.83 & 776.26 & 742.69 & 742.6933 & $\mathbf{7 4 2}$ \\
A-n37-k5 & 100 & 0.81 & 669 & 672.59 & 707.26 & 672.47 & 672.4652 & $\mathbf{6 6 9}$ \\
A-n39-k6 & 100 & 0.88 & 831 & 833.2 & 863.08 & 833.2 & 835.2518 & $\mathbf{8 3 1}$ \\
E-n22-k4 & 6000 & 0.94 & 375 & 375.28 & 388.77 & 375.28 & 375.2798 & $\mathbf{3 7 5}$ \\
E-n33-k4 & 8000 & 0.92 & 835 & 838.72 & 843.1 & 837.67 & 837.9253 & $\mathbf{8 3 5}$ \\
E-n51-k5 & 160 & 0.97 & 521 & 524.94 & 584.64 & 524.61 & 524.6111 & $\mathbf{5 2 1}$ \\
P-n19-k2 & 160 & 0.97 & 212 & 212.66 & 237.9 & 212.66 & 212.6569 & $\mathbf{2 1 2}$ \\
P-n20-k2 & 160 & 0.97 & 216 & 217.42 & 234 & 217.42 & 217.4156 & $\mathbf{2 1 6}$ \\
P-n22-k2 & 160 & 0.96 & 216 & 217.85 & 239.5 & 217.85 & 217.8522 & $\mathbf{2 1 6}$ \\
P-n51-k10 & 80 & 0.97 & 741 & 742.48 & 790.97 & 741.5 & 743.2648 & $\mathbf{7 4 1}$ \\
\hline
\end{tabular}


Table 2

AVONA Table

\begin{tabular}{ccccccc}
\hline Source & SS & df & MS & F & P-value & F crit \\
\hline Columns & 8579.5 & 4 & 2144.88 & 0.03 & 1.00 & 2.56 \\
Error & 3177888 & 50 & 63557.77 & & & \\
\hline Total & 3186468 & 54 & & & & \\
\hline
\end{tabular}

The algorithms of the capacitated VRP instances shown in Table 1 were analyzed using AVONA. Accordingly, we conducted the AVONA with the aim of investigating if the problem size influences the performance of algorithms. There is no prominent difference at the $5 \%$ level. The results from the AVONA are shown in Table 2. Since the value obtained for F does not lie in the critical region at 0.05 , the null hypothesis can be accepted at 5\% level of significance. The performance of algorithms differs with the number of iterations. Consequently, the level of the solution framework may be the source of high-quality result generated by the proposed HCSCROCFO-3Opt. Firstly, there is a creation of high chance of getting a feasible solution by parameter tuning and secondly, the parallel hybrid of CS, CFO and CRO improves the solution quality. Finally, perturbation of the VRP route is generated by the local search with Levy Flight distribution and 3-opt. and the efforts have the possibility of attaining good solutions. Any effort to solve a computational optimization problem using a meta-heuristic is possible with the use of the proposed HCSCROCFO-3Opt parameter tuning methodology, which is one of its major advantages.

\section{Conclusion}

Statistical comparison of the proposed HCSCROCFO-3Opt hybrid's CVRP has been accomplished in this paper. The $\mathrm{CRO}$ algorithm is found as a success in the solution of several benchmark functions (Blum et al., 2010). The CRO does not only escape from the entrapment at a local optimum by itself but also searches for the immediate original solution. Since the CS algorithm retains its stochastic comportment with regard to the Levy Flight distribution, it is possible to attain viable results with the 3-Opt local search. This is because it makes the perturbation effective in examining the global optimum value. The CFO's search strategy mimics physics, which to a greater extent reinforces the speed of the algorithm in getting an average solution. Nevertheless, CS algorithm contains an effective decision mechanism that defines the areas that are in search space and are to be surveyed extensively. The powerful nature of the $\mathrm{CS}$ algorithm is also its strategy to discover solutions in the algorithm as well as managing the discovered nest and egg sources. The search for global optimum value is effective with the perturbation. As such, the proposed hybrid algorithm, the HCSCROCFO-3Opt has been considered to perform better with flexibility in tuning the parameter values, especially when the parameter values are from a maintained set of designs of the experiment's parameter range. The findings are that, there is a flexibility in modifying as well as adjusting based on the problem to be solved. However, it is worth noting that the procedures mentioned above are not able to certify that the tuned parameter provisions will benefit from how the proposed HCSCROCFO-3Opt hybrid unravels the CVRP occurrence. As such, there was an attempt to offer an introductory empirical study to be used to design the experimental sets of parameter values in situations where the proposed algorithm is applied to similar functions. The application of relevant estimation and value calibration method can be adopted to obtain better analysis of the choice of parameter values used in the proposed HCSCROCFO-3Opt hybrid, but that is a study for future work. The algorithm we purpose, named HCSCROCFO-3Opt is based on our previous work (Saeheaw and Charoenchai, 2018), where we integrated the augmented 2-Opt with the cuckoo search (CS), central force optimization (CFO), chemical reaction optimization (CRO) (denoted as CS-CFO-CRO) written in C. Similarly to CS-CFO-CRO, HCSCROCFO-3Opt combines the advantages of each algorithm to form a hybrid algorithm in terms of either computational speed or accuracy. However, differently from CS-CFOCRO, HCSCROCFO-3Opt uses a 3-Opt sequential move to improve searching performance.To validate the algorithm, all codes were written in MATLAB R2018b. As a disclaimer of our work, we do not posit that the proposed HCSCROCFO-3Opt is the most successful in solving all meta-heuristic problems; 
however we can say that it can often outperform the other methods tested in this experiment. Our new study after this will observe the proposed HCSCROCFO-3Opt hybrid in the context of CVRPTW. For now, we would enjoy if other researchers adopt this method in solving their problems.

\section{Acknowledgment}

This work did not receive any specific grant from funding agencies in the public, commercial, or notfor-profit sectors.

\section{References}

Belfiore, P., Tsugunobu, H., \& Yoshizaki, Y., (2008). Chapter 1 Scatter search for vehicle routing problem with time windows and split deliveries, in: Caric, T., Gold, H. (Eds.), Vehicle routing problem.). I-Tech Education and Publishing KG, Vienna, pp. 1-14.

Blazinskas, A., \& Misevicius, A. (2011). combining 2-opt, 3-opt and 4-opt with k-swap-kick perturbations for the traveling salesman problem. Kaunas University of Technology, Department of Multimedia Engineering, Studentu St, 50-401.

Blum, C., Puchinger, J., Raidl, G. R., \& Roli, A. (2010). A brief survey on hybrid metaheuristics. Proceedings of BIOMA, 3-18.

Blum, C., Puchinger, J., Raidl, G.R.,\& Roli, A. (2011). Hybrid metaheuristics in combinatorial optimization: A survey. Applied Soft Computing, 11, 4135-4151.

Blum, C.,\& Roli, A. (2003). Metaheuristics in combinatorial optimization. ACM Computing Surveys, 35, 268308.

Dantzig, G.B.,\& Ramser, J.H. (1959). The truck dispatching problem. Management Science, 6, 80-91.

Formato, R.A. (2007). Central Force Optimization: A new metaheuristic with applications in applied electromagnetics. Progress In Electromagnetics Research, 77, 425-491.

Jin, J.G., Crainic, T.G.,\& Løkketangen, A.G. (2014). A cooperative parallel metaheuristic for the capacitated vehicle routing problem. Computers \& Operations Research, 44, 33-41.

Juan, A.A., Faulin, J., Ruiz, R., Barrios, B.,\& Caballé, S. (2010). The SR-GCWS hybrid algorithm for solving the capacitated vehicle routing problem. Applied Soft Computing, 10, 215-224.

Lam, A.Y.S.,\& Li, V.O.K. (2010). Chemical-reaction-inspired metaheuristic for optimization. IEEE Transactions on Evolutionary Computation, 14, 381-399.

Lam, A. Y., Xu, J., \& Li, V. O. (2010, July). Chemical reaction optimization for population transition in peer-topeer live streaming. In IEEE Congress on Evolutionary Computation (pp. 1-8). IEEE.

Marlow, D. O., Kilby, P., \& Mercer, G. N. (2007, December). The travelling salesman problem in maritime surveillance-techniques, algorithms and analysis. In Proceedings of the international congress on modelling and simulation (pp. 684-690).

Ólafsson, S., (2006). Chapter 21 metaheuristics, in simulation. in: Henderson, S.G., Nelson, B.L. (Eds.), Handbooks in operations research and management science. Elsevier, Amsterdam, pp. 633-654.

Saeheaw, T.,\& Charoenchai, N. (2018). A comparative study among different parallel hybrid artificial intelligent approaches to solve the capacitated vehicle routing problem. International Journal of Bio-Inspired Computation, 11, 171-191.

Urli, T. (2015). Hybrid meta-heuristics for combinatorial optimization [dissertation]. Udine (Italy): Università degli Studi di Udine.

Vidal, M.A., Moreno, P.I., \& Poulin, E. (2012). Genetic diversity and insular colonization of Liolaemus Picts' (Squamata, Liolaeminae) in northwestern Patagonia. Austral Ecology, 37, 67-77.

Yang, X. S. (2010). Nature-inspired metaheuristic algorithms. Luniver press.

Yang, X. S., \& Deb, S. (2009, December). Cuckoo search via Lévy flights. In 2009 World congress on nature \& biologically inspired computing (NaBIC) (pp. 210-214). IEEE.

Zhou, Y., \& Xie, J., Zheng, H. (2013). A hybrid bat algorithm with path relinking for capacitated vehicle routing problem. Mathematical Problems in Engineering, 2013, 1-10.

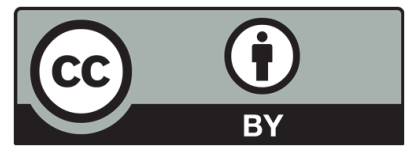

(C) 2020 by the authors; licensee Growing Science, Canada. This is an open access article distributed under the terms and conditions of the Creative Commons Attribution (CCBY) license (http://creativecommons.org/licenses/by/4.0/). 